\title{
Qualitative and Quantitative Analysis of MR Imaging Findings in Patients with Middle Cerebral Artery Stroke Implanted with Mesenchymal Stem Cells
}

\author{
C.P. Wanamaker, S. Fakhran, and L.M. Alhilali
}

\begin{abstract}
BACKGROUND AND PURPOSE: Mesenchymal stem cells have potential as a regenerative therapy in ischemic stroke. We sought to determine MR imaging findings after mesenchymal stem cell implantation in chronic middle cerebral artery infarcts and to compare brain volume changes in patients with mesenchymal stem cells with those in age-matched healthy controls and controls with chronic stable MCA infarcts.
\end{abstract}

MATERIALS AND METHODS: We retrospectively identified 5 patients receiving surgical mesenchymal stem cell implantation to an MCA infarct from January 1, 2005, to July 1, 2013, with MR imaging immediately and 1 year postimplantation. Images at both time points were evaluated for any postimplantation complications. Structural image evaluation using normalization of atrophy software was used to determine volume changes between time points and compare them with those in healthy and age- and sex-matched controls with chronic, stable MCA infarcts by using Kruskal-Wallis and Mann-Whitney $U$ tests.

RESULTS: Susceptibility signal loss and enhancement at the implantation site were seen. No teratoma, tumor, or heterotopia was identified. Volumetric analysis showed a trend toward less overall volume loss after mesenchymal stem cell implantation $(0.736 ; 95 \% \mathrm{Cl}$, $-4.15-5.62)$ compared with that in age- and sex-matched controls with chronic, stable MCA infarcts $(-3.59 ; 95 \% \mathrm{Cl},-12.3$ to $-5.21 ; P=.09)$, with a significantly greater growth-to-loss ratio in infarcted regions (1.30 and 0.78 , respectively, $P=.02)$. A trend toward correlation of growth-to-loss ratio with improvement in physical examination findings was seen $(r=0.856, P=.06)$.

CONCLUSIONS: Postoperative changes consistent with stereotactic implantation were seen, but no teratoma, tumor, or heterotopia was identified. Initial findings suggest a trend toward less volume loss after mesenchymal stem cell implantation compared with that in age- and sex-matched controls with chronic, stable MCA infarcts, with a significantly greater growth-to-loss ratio in the infarcted tissue.

ABBREVIATIONS: \%BVC = percentage brain volume change; GLR = growth-to-loss ratio; MCAI controls = age- and sex-matched controls with chronic, stable MCA infarcts; MSC = mesenchymal stem cells; SIENA = structural image evaluation using normalization of atrophy; $\mathrm{ST}=$ section thickness

$S^{t}$ troke is a leading cause of morbidity and mortality in the United States, ${ }^{1}$ with an estimated $50 \%$ of patients surviving at 90 days demonstrating pronounced long-term disability. ${ }^{2}$ Unfortunately, the only approved drug therapy for ischemic stroke, recombinant tissue plasminogen activator, has a limited therapeutic window. ${ }^{3}$ Given the limited therapeutic options, research into methods to decrease the morbidity associated with ischemic

Received August 20, 2014; accepted after revision December 5.

From the Department of Radiology, Division of Neuroradiology, University of Pittsburgh Medical Center, Pittsburgh, Pennsylvania.

Preliminary data previously presented at: American Society of Neuroradiology Annual Meeting and the Foundation of the ASNR Symposium, May 17-20, 2014; Montreal, Quebec, Canada.

Please address correspondence to Lea M. Alhilali, MD, Department of Radiology, Division of Neuroradiology, University of Pittsburgh Medical Center, 8th Floor, 8 North, Presby South Tower, 200 Lothrop St, Pittsburgh, PA 15213; e-mail: alhilalilm@upmc.edu

三 Indicates article with supplemental on-line table.

http://dx.doi.org/10.3174/ajnr.A4232 stroke has gained pronounced interest. Although rehabilitation has been shown to help functional recovery after stroke, recovery is usually limited. ${ }^{4}$ Cell-based regenerative therapies have been shown to reduce infarct size and improve functional outcomes in animal models of stroke and offer hope of restoring function in patients with fixed neurologic disability following ischemic stroke. $^{5-8}$

Among cell-based therapies, mesenchymal stem cells (MSC) are adult nonhematopoietic pluripotent cells derived from almost all tissues of the body. ${ }^{9}$ These have received considerable attention as a possible regenerative therapy for ischemic stroke due to their multilineage potential, ${ }^{10}$ secretion of trophic factors for neural tissue, ${ }^{11}$ ease of collection, ${ }^{12}$ lack of ethical issues, ${ }^{13}$ efficacy in animals models, ${ }^{14,15}$ and safety in multiple clinical trials. ${ }^{16,17}$

Methods for introducing MSC to an infarct include intravenous, intra-arterial, and intracerebral. ${ }^{18} \mathrm{IV}$ and intra-arterial methods both have certain drawbacks that may be ameliorated by using direct intracerebral implantation. Specifically, IV adminis- 
tration results in first-pass trapping of stem cells in the lung and liver, while intra-arterial administration risks compromise of regional blood flow and MCA occlusion. ${ }^{18}$ Stereotactic implantation is more invasive than either IV or intra-arterial methods. However, it lacks their limitations and offers potentially greater efficacy due to direct delivery of the MSC to infarcted tissues. Intracerebral delivery has been used with success in animal models, demonstrating decreased apoptosis, increased angiogenesis, and neuroprotective effects. ${ }^{19,20}$

Evaluations of the effectiveness of MSC for stroke have mainly focused on changes in clinical function, ${ }^{6,8}$ with only 1 clinical trial evaluating changes on imaging as a possible marker of efficacy following IV administration. ${ }^{7}$ Despite the relative paucity of data on imaging findings after MSC administration, imaging assessment remains important because clinical testing may be undermined by additional neuropsychological factors. For example, symptoms related to comorbidities, such as depression, may clinically mimic persistent neurologic dysfunction, while poor motivation and ulterior motives may undermine the accuracy of clinical tests. However, if concomitant imaging findings can be matched with clinical testing, this matching may provide a quantitative anatomic biomarker to confirm clinical findings.

A prior clinical trial has evaluated changes in infarct T2 hyperintensity after MSC intravenous administration ${ }^{7}$; however, no control patients were evaluated, and it remains unclear whether observed changes in T2 hyperintensity merely represented the natural history of the infarcted tissue or response to MSC. The purpose of our study was to determine MR imaging findings after MSC implantation in patients with chronic MCA infarcts and to compare global and regional brain volume changes in patients with MSC implants with age-matched healthy controls and controls with chronic stable MCA infarcts (MCAI controls).

\section{MATERIALS AND METHODS \\ Patient Selection and Image Acquisition}

Our institutional review board approved this study, with a waiver of informed consent. All studies included were performed as part of a research protocol, and results were retrospectively reviewed.

We searched our electronic medical record to identify MR imaging studies performed on patients who had undergone implantation of MSC for MCA infarction. Stem cell implantation is performed surgically at our institution as part of a research protocol on a small number of study patients by using a Leksell Coordinate Frame G stereotactic head frame (Elekta Instruments, Stockholm, Sweden). We make 3 target selections: an anterior, mid, and posterior target for the medial wall of each infarct, regardless of infarct size. The xyz coordinates are calculated, and stem cells are verified and loaded. The 3 targets are then used, and $20 \mu \mathrm{L}$ is injected to each target point in a single burr-hole trajectory, with small volumes injected each time for several minutes.

MR imaging reports from January 1, 2005, to July 1, 2013, were searched by using the keywords "stem cell." Inclusion criteria were the following: age, 18-75 years; MCA ischemic infarct with no prior infarcts; 6 - to 60-month intervals between the infarct and imaging; no further improvement from physical therapy after at least 6 months; initial MR imaging within 1 day of MSC implantation; and follow-up MR imaging 10-14 months after implanta- tion. Exclusion criteria were the following: sufficient motion degradation to preclude volumetric analysis as determined by a neuroradiologist with $>3$ years of experience with quantitative imaging analysis, lack of follow-up imaging, any other major neurologic disease, any malignancy except squamous or basal cell carcinoma of the skin, contraindication to MR imaging, pregnancy, or lactation. Demographic data recorded included patient age and sex. The location of the infarct, infarct etiology, and physical examination findings initially and $>10$ months post-MSC implantation by either a primary care physician or neurologist were likewise recorded.

Patient recovery was graded by a recovery score, with a point system determined as follows:

1) We recorded preimplantation deficits in the following categories: facial sensation (1) and strength (2), upper extremity sensation (3) and strength (4), lower extremity sensation (5) and strength (6), visual acuity (7), and language (8).

2) If there was a deficit in a given category that demonstrated improvement, then the patient received a score of 1 in that category. If there was no change, the score was zero. If there was deterioration, the score was -1 . If there was no initial deficit in a category, the patient received no score for the given category.

3) The total number of points for all categories was summed and divided by the total number of categories scored. The resulting number was termed the "total recovery score."

Age- and sex-matched controls were obtained by using an individual matching method in which each control was within 5 years of age of the individual matched subject. Controls were found by searching the electronic medical record for MR imaging examinations by using the keywords "unremarkable" and "within normal limits." Controls were excluded if there was any infarct detected on imaging as determined by a fellowship-trained radiologist, any evidence of vasculopathy, a history of any other major neurologic disease, lack of follow-up imaging within 10-14 months, interval infarct, hemorrhage or other neurologic disease process, or sufficient motion degradation to preclude volumetric analysis. Reasons for MR imaging examinations in controls were memory loss (3 patients) and dizziness (2 patients).

Age- and sex-matched control subjects with chronic, stable MCA encephalomalacia (referred to as MCAI controls) were also obtained by using an individual matching method in which each MCAI control was within 5 years of age of the individual matched stem-cell subject. MCAI controls were found by searching the electronic medical record for MR imaging examinations by using the keywords "middle cerebral artery," "stroke," "infarct," and "encephalomalacia." Inclusion criteria for MCAI controls were the following: ischemic middle cerebral artery infarct between 6 and 60 months of age based on review of the electronic medical record and follow-up imaging between 10 and 14 months of the initial MR imaging. MCAI controls were excluded if there was sufficient motion degradation to preclude volumetric analysis. Comparison of the time interval between initial and follow-up imaging between subjects with MSC implants and the MCAI controls was determined with the Mann Whitney $U$ test.

All subjects and controls underwent MR imaging at 2 time points (initial imaging and 10-14 months after initial imaging) on a $1.5 \mathrm{~T}$ system (Signa; GE Healthcare, Milwaukee, Wisconsin) 
with a standard head coil. Sequences included sagittal and axial T1-weighted (TR, 600 ms; TE, minimum; section thickness [ST], $5 \mathrm{~mm}$; NEX, 1), fast spin-echo axial proton-attenuation (TR, 2000-2500 ms; TE, minimum; ST, 5 mm; NEX, 1), T2-weighted (TR, 2000-2500 ms; TE, 84-102 ms; ST, 5 mm; NEX, 1), fluidattenuated inversion-recovery (TR, 9000-10,000 ms; TE, $149 \mathrm{~ms}$; TI, $2200 \mathrm{~ms}$ ), and diffusion-weighted (single-shot echo-planar; TR, 10,000 ms; TE, minimum; ST, 5 mm; matrix, 128). Either T2* gradient recalled-echo (TR/TE/NEX/flip angle, $4400 \mathrm{~ms} / 21 \mathrm{~ms} / 1 /$ $90^{\circ}$; ST, $3 \mathrm{~mm}$ ), susceptibility-weighted (TR/TE/NEX/flip angle, $37 \mathrm{~ms} / 23 \mathrm{~ms} / 1 / 15^{\circ}$; ST, $2.4 \mathrm{~mm}$ ), or echo-spoiled gradient-echo imaging (TR/TE/NEX, 25 ms/3 ms/1; ST, 1.0 mm; matrix, $256 \times$ 256) was performed. The FOV ranged from 200 to $240 \mathrm{~mm}$. Contrast-enhanced echo-spoiled gradient-echo images were obtained with $0.1-\mathrm{mmol} / \mathrm{kg}$ gadolinium-based contrast material (gadobenate dimeglumine, MultiHance; Bracco Diagnostics, Princeton, New Jersey) by using parameters as described above, performed immediately ( $<1$ minute) after contrast administration.

\section{Evaluation for Imaging Findings Associated with MSC Implantation}

MR images from both imaging time points in all patients with MSC implants were reviewed by 2 fellowship-trained neuroradiologists (S.F., L.M.A.) for the following findings at both imaging time points: 1) interval infarction or hemorrhage remote from the MSC implantation site, 2) any neoplasm-especially teratoma-or heterotopia, 3) qualitative evaluation of any edema (mild, moderate, severe), and 4) the presence or absence of any enhancement. Differences were resolved by consensus.

\section{Quantitative Volumetric Analysis}

Percentage Brain Volume Change. Comparison of the percentage of brain volume change ( $\% \mathrm{BVC}$ ) between the 2 time points for patients with MSC implants, controls, and MCAI controls was performed with the Structural Image Evaluation using Normalization of Atrophy (SIENA) software (fMRI of the Brain Software Library, Version 3.2 software suite; http://www.fmrib.ox.ac.uk/ fsl). ${ }^{21,22}$ SIENA determines the \%BVC in the following steps: 1 ) The brain is segmented from nonbrain tissue, and the skull surface is estimated from the anatomic images; 2) this segmentation is then used to coregister the 2 images at each time point and normalize for changes in geometric shape; and 3) local changes in brain volume are determined on the basis of movement associated with image edges (Fig 1A,-B). Calculated volumetric changes are presented as \%BVC between the 2 imaging time points.

Although several automated methods are available for measurements of brain volume change-SIENA, Unified Segmentation (SPM5; http://www.fil.ion.ucl.ac.uk/spm/software/spm5), and k-Nearest Neighbor-based probabilistic segmentation being the most popular-SIENA was chosen due to its previously reported high accuracy in the evaluation of longitudinal changes in brain volume, ${ }^{22}$ and its superior performance in direct comparisons. ${ }^{23}$ The principal limitation to all of these methods is variability in results when multiple scanners are used, particularly with differing field strengths and at different centers. ${ }^{23,24}$

Comparison of \%BVC among the 3 groups (patients with MSC implants, controls, and MCAI controls) was performed with

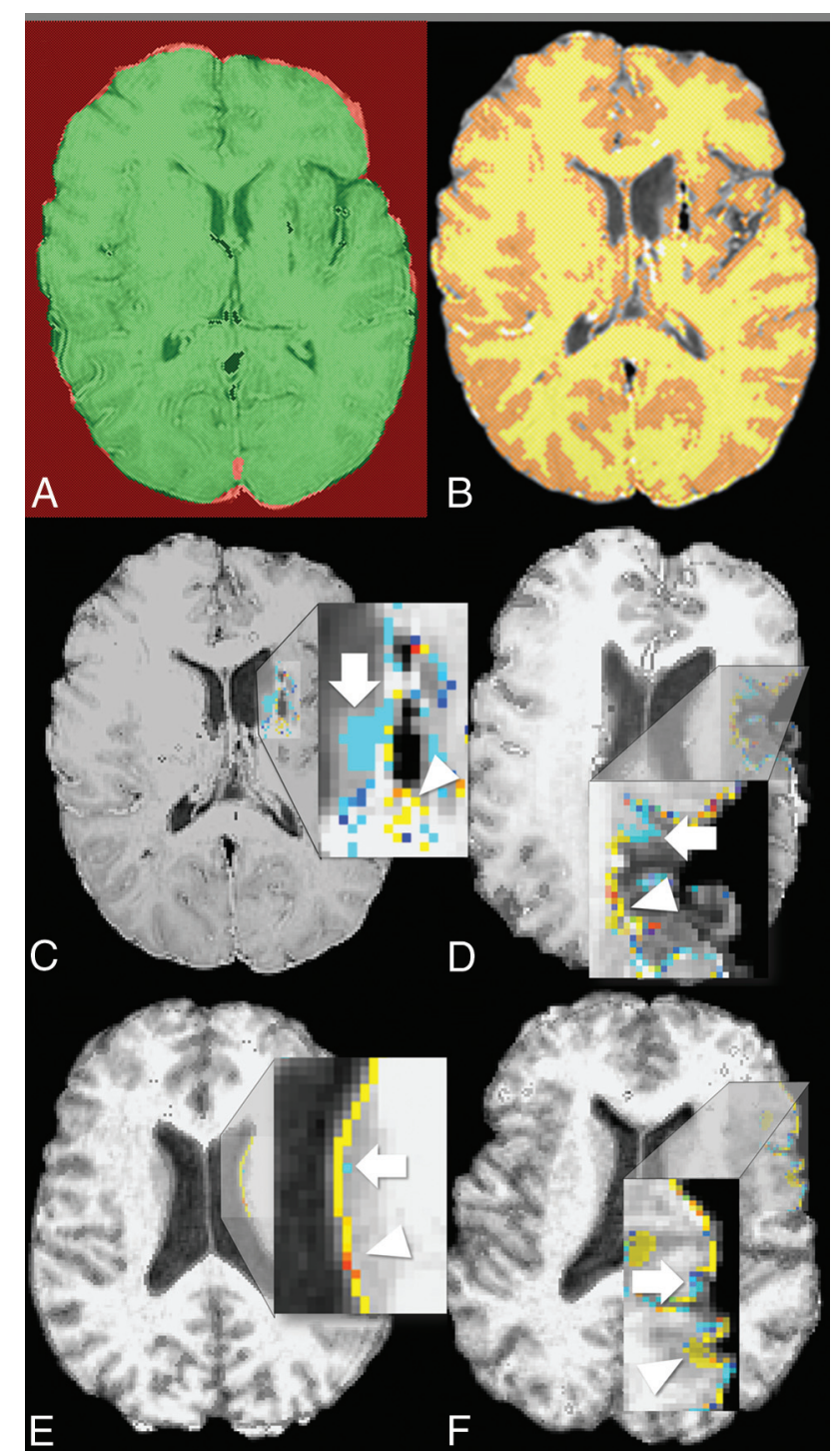

FIG 1. Calculation of percentage brain volume change and growthto-loss ratio. A, Brain and skull images are initially each extracted from whole-head input data from the 2 separate time points for each individual subject. The 2 images ( 1 at each time point) are aligned to each other by using the skull images to constrain registration scaling at each time point. Red demonstrates the common FOV. Green shows the intersection of the 2 standard space brain masks. B, Tissue-type segmentation is used to define the brain/nonbrain boundary. The perpendicular edge displacement at the brain/nonbrain boundary between the 2 time points is estimated, and the mean edge displacement is converted in the estimate of the percentage brain volume change between the 2 points. $C$ and $D$, The growth-to-loss ratio is calculated in 2 different patients with mesenchymal stem cell implants from the color-rendered image of edge motion superimposed on a half-way image, created by aligning the 2 brain images from the 2 time points to each other by using the skull images to constrain the registration scaling. Red-yellow indicates brain volume increase (white arrowhead), while blue-light blue indicates brain volume decrease ("atrophy") (white arrow). Regions of growth (red-yellow) are seen to be predominantly at the medial margin of the infarct, where the stem cells are implanted. $E$ and $F$, Comparable periventricular and cortical regions are shown in healthy controls, demonstrating more red-yellow voxels in similar areas in healthy controls compared with the infarcted regions in patients with MSC implants. The GLR is calculated by tabulating the ratio of the total number of red-yellow voxels (growth areas) to the total number of blue-light blue voxels (areas of volume loss) in the region of the infarct. 
a Kruskal-Wallis test, followed by pair-wise analysis by using a Mann-Whitney $U$ test.

Regional Brain Volume Change. Regional brain volume changes in regions of infarction were assessed for patients with MSC implants and MCAI controls by using color maps of voxelwise change in brain volume generated by SIENA. Voxels demarcating a region of brain volume loss were color-coded blue or light blue, while voxels indicating a region of brain volume increase were color-coded yellow or red. Voxels in which volume remained unchanged were color-coded gray. The total number of blue-light blue and yellow/red voxels for each infarct was separately tabulated by a fellowship-trained neuroradiologist with $>3$ years of image analysis experience (L.M.A.), blinded to the clinical findings. The ratio of yellow/red to blue-light blue voxels was calculated to create a growth-to-loss ratio (GLR), to compare volume changes in infarcted tissue between the 2 groups, controlling for infarct size (Fig $1 C,-D$ ). Comparison of the GLR between patients with MSC implants and MCAI controls was performed with a Mann-Whitney $U$ test. Correlation of GLR with the total recovery score was performed with the Pearson correlation coefficient.

\section{RESULTS}

\section{Patient Selection and Image Acquisition}

Six patients underwent MSC implantation. One patient was excluded because appropriate follow-up imaging had not been performed. The remaining 5 patients were included in this study ( 3 men, 2 women; mean age, 62 years; range, 53-77 years). All patients underwent initial MR imaging within 1 day of MSC implantation and had a follow-up MR imaging 12 months after the initial imaging. No patients were lost to follow-up. The average time interval from stroke to MSC implantation was 23 months (range, 6-36 months). The patient demographics, etiology of the infarcts, initial physical examination findings, and physical examination findings post-MSC implantation are shown in the On-line Table.

Five age- and sex-matched controls were included ( 3 men, 2 women; mean age, 59 years; range, $51-76$ years). The average time between imaging was 10.4 months (range, 10-11 months).

Five age- and sex-matched MCAI controls were included (3 men, 2 women; mean age, 64 years; range, $48-82$ years). The average time between imaging was 12.4 months (range, 11-14 months). No significant difference was seen in the imaging time interval between MCAI controls and patients with MSC implants $(P=.67, U=10)$.

\section{Evaluation for Imaging Findings Associated with Stem Cell Implantation}

The immediate postprocedural findings corresponded well with known complications post-stereotactic biopsy. ${ }^{25}$ One patient had a small subdural collection immediately post-MSC implantation deep to the burr-hole site measuring approximately $6 \mathrm{~mm}$. No new infarct was identified in any patient.

Three patients had mild edema along the operative tract, while 2 had moderate edema. No edema persisted on follow-up imaging. All patients had enhancement at the implantation site, and 2

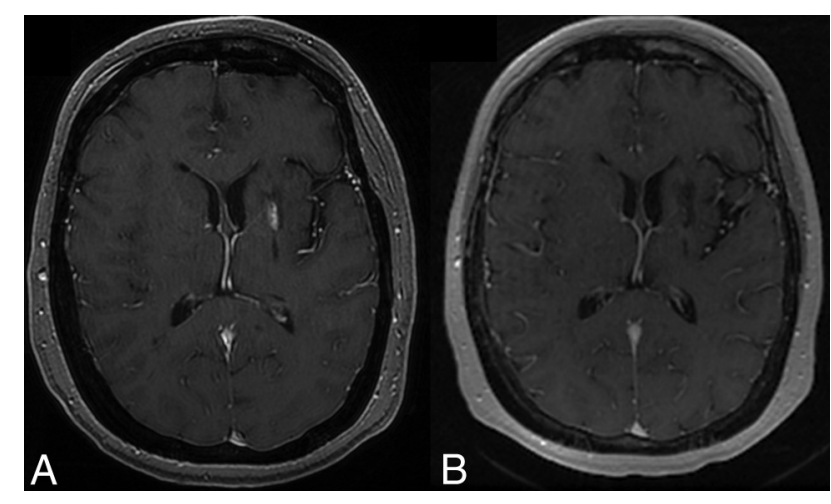

FIG 2. Resolution of enhancement at the implantation site on follow-up imaging. Postcontrast axial echo-spoiled gradient-echo sequence images in a 54-year-old woman status post stem cell implantation demonstrate initial enhancement at the implantation site immediately postoperatively $(A)$, which resolved on 1-year follow-up imaging $(B)$.

Kruskal-Wallis analysis of \%BVC among healthy controls, patients with stem cell (MSC) implantation, and MCAI controls

\begin{tabular}{lccc}
\hline & $\begin{array}{c}\text { Healthy } \\
\text { Controls }\end{array}$ & $\begin{array}{c}\text { MSC } \\
\text { Implantation }\end{array}$ & $\begin{array}{c}\text { MCAI } \\
\text { Controls }\end{array}$ \\
\hline \% BVC & 2.00 & 0.736 & -3.59 \\
$95 \% \mathrm{Cl}$ & $-1.58-5.58$ & $-4.15-5.62$ & -12.3 to -5.21 \\
$\begin{array}{c}P \text { value compared with } \\
\text { healthy controls }\end{array}$ & $\mathrm{NA}$ & .38 & .06 \\
$\begin{array}{c}P \text { value compared with } \\
\text { patients with MSC }\end{array}$ & .38 & $\mathrm{NA}$ & .09 \\
$\begin{array}{c}P \text { value compared with } \\
\text { MCAl controls }\end{array}$ & .06 & .09 & $\mathrm{NA}$ \\
\hline
\end{tabular}

Note:-NA indicates not applicable.

${ }^{a} P$ values from a post hoc pair-wise analysis using the Mann-Whitney $U$ test of the Kruskal-Wallis analysis results.

had enhancement along the implantation tract. No enhancement persisted on follow-up imaging (Fig 2). All patients had punctate susceptibility signal loss along both the implantation tract and site, suggestive of hemosiderin staining, which persisted at follow-up imaging.

No neoplasm or sign of heterotopia was detected in any patient.

\section{Quantitative Volumetric Analysis}

\%BVC. Mean \%BVC among controls, MCAI controls, and subjects with MSC implants (Table) was significantly different $(P=$ .046 , H value $=6.14)$. Post hoc analysis found that MCAI controls trended toward greater volume loss than healthy controls $(P=$ .06). No significant difference in volume loss was seen between patients with MSC implants and healthy controls $(P=.14)$. However, patients with MSC implants trended toward less volume loss compared with MCAI controls $(P=.09)$.

Regional Brain Volume Change. The GLR for patients with MSC implants (mean, 1.30; range, 0.92-1.42) was significantly higher than that for MCAI controls (mean, 0.78; range, 0.56-1.05; $P=$ $.02)$. There was a trend toward correlation of GLR with a higher total recovery score $(P=.06, r=0.856)$. The total recovery score did not correlate with time from stroke to implantation $(P=.42$, $r=-0.477)$. 


\section{DISCUSSION}

Implantation of MSC results in typical imaging changes seen after stereotactic intervention, with an additional finding of transient enhancement at the implantation site. No complications unique to MSC, such as a teratoma or heterotopia, were detected. Most important, MSC implantation after stroke resulted in a trend toward overall less volume loss and more regions of growth at the infarct site compared with controls with infarcts who did not receive MSC.

While currently use of MSC is essentially limited to clinical trials, the growing body of evidence of their safety in humans ${ }^{6-8}$ suggests that imaging of patients who have received MSC will only become more common in the future. It is, therefore, important for the interpreting radiologist to be aware of the expected imaging findings and their time course. Previous studies have looked at changes in the appearance of the treated stroke after implantation but have not evaluated changes related to the implantation itself. ${ }^{7}$ Our study evaluated MSC administered via stereotactic implantation. While enhancement and edema may be seen immediately after any stereotactic intervention, ${ }^{25}$ these findings resolved on follow-up imaging in all our patients. Therefore, on the basis of our preliminary findings, persistent enhancement or edema may possibly indicate a secondary process, though further studies are needed to confirm the natural history of the imaging findings.

The most feared complications associated with MSC implantation, including teratoma formation and heterotopias, were not seen in our patients, similar to findings in previous studies. ${ }^{26} \mathrm{Al}-$ though our follow-up was only 1 year, previous animal experiments have suggested that neoplastic complications typically appear within 1 month of treatment. ${ }^{27,28}$ Similarly, although MSC may induce an inflammatory reaction, ${ }^{29,30}$ no progressive enhancement or edema was seen in our cohort. This may be because MSC also have immunomodulatory properties ${ }^{31}$ that may suppress an inflammatory reaction. This finding bodes well for the efficacy of MSC in the treatment of stroke because the immunosuppressive properties of MSC are key to their regenerative capacity. Immune/inflammatory cells play a key role in tissue injury in patients with stroke. ${ }^{14}$ MSC combats this inflammatory cascade and promotes tissue regeneration/repair by secreting growth factors suppressing these inflammatory cytokines, resulting in enhanced angiogenesis, decreased leukocyte transmigration, and greater stem cell differentiation. ${ }^{32}$ Larger studies to confirm lack of an inflammatory response should be a goal of future research.

Our preliminary findings support the effectiveness of MSC in promoting tissue regeneration through these described mechanisms. While our cohort was small, we found a trend toward less brain volume loss and significantly greater growth at the infarct site in the transplanted cohort compared with the MCAI cohort. This finding is encouraging because the therapeutic action of the implanted MSC is believed to be related to the secretion of trophic factors that would stimulate neuronal survival and differentiation of native stem cells. ${ }^{33}$ Notably, this greater GLR after implantation trended toward a correlation with improved clinical findings in our patients, suggesting that the imaging findings may be used as a marker of clinical improvement that can assist in further clinical trials. Previous stroke studies have correlated the volume of diffusion abnormality ${ }^{34}$ or the final infarct volume ${ }^{35}$ with clin- ical outcomes after intervention with thrombolysis, and perhaps the GLR may serve as a similar predictor of clinical outcome after intervention with MSC. However, future studies are needed to confirm this trend toward improved clinical function with greater growth on imaging.

Our assessment of MR imaging findings after MSC implantation is limited by our small number of patients and short follow-up interval. However, larger cohorts are difficult to amass, given the current relatively limited use of stem cells. It will also be important to correlate imaging findings with objective stroke scales before and after implantation, to quantify any clinical benefit. Additional imaging of white matter tracts would be another avenue to explore the effects of MSC implantation on new white matter tracts and brain plasticity.

\section{CONCLUSIONS}

Initial findings after MSC implantation demonstrate expected postprocedural changes after a stereotactic procedure and no unique complications such as teratoma, tumor, or heterotopia. A significantly greater growth-to-loss ratio in infarcted tissue was seen in patients receiving MSC compared with patients who did not receive MSC, which trended toward a correlation with an improvement in physical examination findings.

\section{REFERENCES}

1. Roger VL, Go AS, Lloyd-Jones DM, et al; for the American Heart Association Statistics Committee and Stroke Statistics Subcommittee. Heart disease and stroke statistics: 2012 update-a report from the American Heart Association. Circulation 2012;125:e2-220

2. Johnston SC, Mendis S, Mathers CD. Global variation in stroke burden and mortality: estimates from monitoring, surveillance, and modelling. Lancet Neurol 2009;8:345-54

3. Adeoye $\mathrm{O}$, Hornung R, Khatri P, et al. Recombinant tissue-type plasminogen activator use for ischemic stroke in the United States: a doubling of treatment rates over the course of $\mathbf{5}$ years. Stroke 2011;42:1952-55

4. Hong KS, Ali LK, Selco SL, et al. Weighting components of composite end points in clinical trials: an approach using disability-adjusted life-years. Stroke 2011;42:1722-29

5. Friedrich MA, Martins MP, Araújo MD, et al. Intra-arterial infusion of autologous bone marrow mononuclear cells in patients with moderate to severe middle cerebral artery acute ischemic stroke. Cell Transplant 2012;21(suppl 1):S13-21

6. Lee JS, Hong JM, Moon GJ, et al. A long-term follow-up study of intravenous autologous mesenchymal stem cell transplantation in patients with ischemic stroke. Stem Cells 2010;28:1099-106

7. Honmou $\mathrm{O}$, Houkin $\mathrm{K}$, Matsunaga $\mathrm{T}$, et al. Intravenous administration of auto serum-expanded autologous mesenchymal stem cells in stroke. Brain 2011;134(pt 6):1790-807

8. Bhasin A, Srivastava MV, Mohanty S, et al. Stem cell therapy: a clinical trial of stroke. Clin Neurol Neurosurg 2013;115:1003-08

9. Glover LE, Tajiri N, Weinbren NL, et al. A step-up approach for cell therapy in stroke: translational hurdles of bone marrow-derived stem cells. Transl Stroke Res 2012;3:90-98

10. Mezey E, Chandross KJ, Harta G, et al. Turning blood into brain: cells bearing neuronal antigens generated in vivo from bone marrow. Science 2000;290:1779-82

11. Phinney DG, Prockop DJ. Concise review: mesenchymal stem/multipotent stromal cells: the state of transdifferentiation and modes of tissue repair-current views. Stem Cells 2007;25:2896-902

12. Mohal JS, Tailor HD, Khan WS. Sources of adult mesenchymal stem cells and their applicability for musculoskeletal applications. Curr Stem Cell Res Ther 2012;7:103-09 
13. Ikegame Y. Among mesenchymal stem cells: for the best therapy after ischemic stroke. Stem Cell Res Ther 2013;4:9

14. Tatarishvili J, Oki K, Monni E, et al. Human induced pluripotent stem cells improve recovery in stroke-injured aged rats. Restor $\mathrm{Neu}$ rol Neurosci 2014;32:547-58

15. Tornero D, Wattananit S, Grønning Madsen M, et al. Human induced pluripotent stem cell-derived cortical neurons integrate in stroke-injured cortex and improve functional recovery. Brain 2013; 136(pt 12):3561-77

16. Burns TC, Steinberg GK. Stem cells and stroke: opportunities, challenges and strategies. Expert Opin Biol Ther 2011;11:447-61

17. Bang OY, Lee JS, Lee PH, et al. Autologous mesenchymal stem cell transplantation in stroke patients. Ann Neurol 2005;57:874-82

18. Hao L, Zou Z, Tian H, et al. Stem cell-based therapies for ischemic stroke. Biomed Res Int 2014;2014:468748

19. Bao X, Feng M, Wei J, et al. Transplantation of Flk-1+ human bone marrow-derived mesenchymal stem cells promotes angiogenesis and neurogenesis after cerebral ischemia in rats. Eur J Neurosci 2011;34:87-98

20. Li J, Zhu H, Liu Y, et al. Human mesenchymal stem cell transplantation protects against cerebral ischemic injury and upregulates interleukin-10 expression in Macaca fascicularis. Brain Res 2010; 1334:65-72

21. Smith SM, De Stefano N, Jenkinson M, et al. Normalized accurate measurement of longitudinal brain change. J Comput Assist Tomogr 2001;25:466-75

22. Smith SM, Zhang Y, Jenkinson M, et al. Accurate, robust, and automated longitudinal and cross-sectional brain change analysis. $\mathrm{Neu}$ roimage 2002;17:479-89

23. de Bresser J, Portegies M, Leemans A, et al. A comparion of MR based segmentation methods for measuring brain atrophy progression. Neuroimage 2011;54:760-68

24. Durand-Dubief F, Belaroussi B, Armspach JP, et al. Reliability of longitudinal brain volume loss measurements between 2 sites in patients with multiple sclerosis: comparison of 7 quantification techniques. AJNR Am J Neuroradiol 2012;33:1918-24

25. Hall WA. The safety and efficacy of stereotactic biopsy for intracranial lesions. Cancer 1998;82:1749-55

26. Eckert MA, Vu Q, Xie K, et al. Evidence for high translational potential of mesenchymal stromal cell therapy to improve recovery from ischemic stroke. J Cereb Blood Flow Metab 2013;33:1322-34

27. Aldahmash A, Atteya M, Elsafadi M, et al. Teratoma formation in immunocompetent mice after syngeneic and allogeneic implantation of germline capable mouse embryonic stem cells. Asian Pac J Cancer Prev 2013;14:5705-11

28. Yamamoto M, Cui L, Johkura K, et al. Branching ducts similar to mesonephric ducts or ureteric buds in teratomas originating from mouse embryonic stem cells. Am J Physiol Renal Physiol 2006; 290:F52-60

29. Inoue S, Popp FC, Koehl GE, et al. Immunomodulatory effects of mesenchymal stem cells in a rat organ transplant model. Transplantation 2006;81:1589-95

30. François M, Romieu-Mourez R, Stock-Martineau S, et al. Mesenchymal stromal cells cross-present soluble exogenous antigens as part of their antigen-presenting cell properties. Blood 2009;114:2632-38

31. Ren G, Su J, Zhang L, et al. Species variation in the mechanisms of mesenchymal stem cell-mediated immunosuppression. Stem Cells 2009;27:1954-62

32. Ma S, Xie N, Li W, et al. Immunobiology of mesenchymal stem cells. Cell Death Differ 2014;21:216-25

33. Liu X, Ye R, Yan T, et al. Cell based therapies for ischemic stroke: from basic science to bedside. Prog Neurobiol 2014;115:92-115

34. Turc G, Aguettaz P, Ponchelle-Dequatre N, et al. External validation of the MRI-DRAGON score: early prediction of stroke outcome after intravenous thrombolysis. PLoS One 2014;9:e99164

35. Yoo AJ, Chaudhry ZA, Nogueira RG, et al. Infarct volume is a pivotal biomarker after intra-arterial stroke therapy. Stroke 2012;43: 1323-30 